\title{
Completing the Corticofugal Loop: A Visual Role for the Corticogeniculate Type 1 Metabotropic Glutamate Receptor
}

\author{
Casto Rivadulla, Luis M. Martínez, Carmen Varela, and Javier Cudeiro \\ Neuroscience and Motor Control Group (NEUROcom), Facultad de Ciencias de la Salud (Departamento de Medicina) \\ and Instituto Nacional de Educacion Fisica de Galicia, Campus de Oza, 15006 A Coruña, Spain
}

\begin{abstract}
The way in which the brain deals with sensory information relies not only on feedforward processing of signals from the periphery but also on feedback inputs. This is the case of the massive projection back from layer 6 in the visual cortex to the thalamus, for which, despite being the greatest single source of synaptic contacts, the functional role still remains unclear. In the cat lateral geniculate nucleus, part of this cortical feedback is mediated by type 1 metabotropic glutamate receptors (mGluR1s), which are exclusively located on distal segments of the relay-cell dendrites. Here we show that in adult cats the cortex uses a synaptic drive mediated by these receptors
\end{abstract}

In the early visual pathway, information travels from the lateral geniculate nucleus (LGN) to the primary visual cortex. At this level, the relay of visual information is regulated by a network of synaptic connections, including modulatory projections from brainstem nuclei (Ahlsen and Lo, 1982; De Lima and Singer, 1987a,b; Steriade et al., 1988; Bickford et al., 1993; Uhlrich et al., 1995; Cudeiro and Rivadulla, 1999), GABAergic inputs from local interneurons and perigeniculate neurons (Montero, 1986, 1987), and a feedback projection from the primary visual cortex using both ionotropic and metabotropic [metabotropic glutamate receptor (mGluR)] excitatory amino acid receptors (Montero and Wenthold, 1989; Scharfman et al., 1990; McCormick and von Krosigk, 1992). Two characteristics differentiate the cortical feedback from the other modulatory inputs: first, the number of fibers largely outnumbers any other projection (Van Horn et al., 2000), and second, the retinotopy of the pathway is preserved precisely (Updyke, 1975, 1977), making the visual cortex an ideal candidate to exert a point-to-point control onto the flow of information traveling through the LGN (Murphy et al., 1999). Previous investigations over several decades (Singer, 1977) have shown that corticofugal projections modify the excitability of thalamic neurons. Growing evidence favors an active role of feedback connections in visual processing, influencing properties of visual stimulus such as, for example, orientation and direction of movement (Sillito et al., 1993; Murphy et al., 1999) and contributing to the generation of the sensitivity of a cell to the length of a moving contour, the so-called length tuning (LT) (Murphy and Sillito, 1987), which was first observed in the cortex (Hubel and Wiesel, 1965).

\footnotetext{
Received Dec. 7, 2001; revised Jan. 22, 2002; accepted Jan. 23, 2002.

This work was supported by Comision Interministerial de Ciencia y Tecnologia (PB1998-0179), Spain. We thank K. L. Grieve for helpf ul comments and corrections on this manuscript.

Correspondence should be addressed to Javier Cudeiro, Departamento de Medicina, Campus de Oza, 15006 A Coruña, Spain. E-mail: jcud@udc.es.

Copyright (C) 2002 Society for Neuroscience 0270-6474/02/222956-07\$15.00/0
}

(mGluR1) specifically to enhance the excitatory center of the thalamic receptive field. Moreover the effect is maximum in response to those stimuli that effectively drive cortical cells, and importantly, it does not affect the spatiotemporal structure of the thalamic receptive field. Therefore, cortex, by closing this corticofugal "loop," is able to increase the gain of its thalamic input within a focal spatial window, selecting key features of the incoming signal.

Key words: LGN; visual cortex; corticothalamic; metabotropic receptors; feedback modulation; layer 6

In the cat, the LGN represents an excellent model for the study of the role exerted by corticofugal axons, because retinal and cortical inputs are segregated on relay cells, with retinal afferents contacting proximal dendrites and cortical terminals contacting distal dendrites (Wilson et al., 1984; Erisir et al., 1997). In addition, there is also segregation of the distribution of subtypes of the metabotropic glutamate receptors that matches this synaptic input distribution, with type 1 mGluRs (mGluR1s) found in the distal dendrites of relay cells (associated with cortical terminals) and mGluR5s being in the synaptic terminal of interneurons, coupled to retinal inputs (Godwin et al., 1996) (see Fig. 1).

To study the role of corticogeniculate inputs in visual processing, we extracellularly recorded the visual responses of LGN cells before, during, and after the iontophoretic ejection of $(+) 2$ methyl-4-carboxyphenylglycine (LY367385), a highly specific mGluR1 antagonist (Clark et al., 1997; Salt et al., 1999; Marino et al., 2001). We used two sets of visual stimuli: static stimuli (flashing spots), considered to drive LGN neurons strongly but not to activate corticothalamic cells, and moving patterns (bars or gratings), considered to better activate corticothalamic cells. To dissect out specific effects from global changes in firing rate caused by changes in inhibitory balance, we have compared responses obtained during mGluR1 blockade with those recorded during GABA ejection. Our results indicate that cortical feedback mediated by mGluR1 receptors is likely to enhance thalamic center-surround antagonism, with major consequences for properties like LT, but it does not affect the spatiotemporal structure of the thalamic receptive field measured with static stimuli.

Parts of these results have been published previously in abstract form (Varela et al., 2000).

\section{MATERIALS AND METHODS}

Adult cats, $2.5-3.5 \mathrm{~kg}$, were anesthetized with halothane $(0.1-1 \%$ in $70 \%$ $\left.\mathrm{N}_{2} \mathrm{O} / 30 \% \quad \mathrm{O}_{2}\right)$ and paralyzed with gallamine triethiodide (10 $\mathrm{mg} \cdot \mathrm{kg}^{-1} \cdot \mathrm{hr}^{-1}$ ). Full details are given in Cudeiro et al. (2000). To further reduce possible eye movement artifacts, posts were fixed to the 
sclera. The procedures conformed to the Spanish Physiology Society and the International Council for Laboratory Animal Science and the European Union (statute 86/809).

Extracellular recording and iontophoresis. All of our observations were made in the A laminas of the LGN. Multibarreled pipettes (three to five barrels) were used for extracellular recording and iontophoretic ejection of drugs. Waveforms and time stamps were stored (Plexon Inc.), and we carefully performed off-line spike sorting to assess the isolation of the selected spike and recording stability. The barrels were filled with $\mathrm{NaCl}$ (3 $\mathrm{M})$ for recording, and an aqueous solution of $50 \mathrm{~mm} \mathrm{LY367385,} \mathrm{pH} 8$. Ach (1 M, pH 5) was included in the pipette for identifying relay cells on the basis of its excitatory effect (McCormick and Prince, 1987). Ejection currents were in the range of 50-80 nA; this range was selected on the basis of initial qualitative observations. When not in use, each drug barrel was subject to a constant retention current of 5-25 nA of appropriate polarity. When a cell was isolated, we measured its response to the visual stimuli and repeated the same protocol during ejection of LY367385 and once more after a period of recovery. Only cells that showed recovery of responses $>80 \%$ of control values were included in this study.

Visual stimulation. Computer-controlled visual stimuli (Lohmann Research Equipment) were presented monocularly on a monitor with a mean luminance of $14 \mathrm{~cd} / \mathrm{m}^{2}$ at a contrast of 0.7 , refresh rate $128 \mathrm{~Hz}$. Visual stimuli consisted of sinusoidal drifting wave gratings, spots of different size flashing on the receptive field (RF), and moving bars of different length. The spatiotemporal properties of the stimuli were optimally set for each cell. Stimuli were presented in pseudorandom order and included two blank presentations to obtain spontaneous activity. Responses were collected for the entire duration of the stimulus and were averaged from 8 to 15 presentations. For drifting gratings, each presentation included 10 cycles of the stimulus. Receptive fields were mapped with moving bars and in 10 cells by reverse correlation with sparse noise (Jones and Palmer, 1987; Hirsch et al., 1998). The sparse noise protocol consisted of light or dark squares flashed singly for $40 \mathrm{msec}$ in pseudorandom order, 30 times each, on a $20 \times 20$ grid (square size, $0.4-1^{\circ}$; contrast ranged from 80 to $100 \%$ ). RFs are plotted as pixel maps where the magnitude of the response is coded in a grayscale. All maps are constructed for just one polarity of the stimulus. LT index was calculated as (spikes evoked by optimal stimulus - spikes evoked by non-optimal)/ spikes evoked by optimal. Number of spikes was calculated from the accumulated count in all the bins constituting the response area in the peristimulus time histograms (PSTHs), after subtraction of the background discharge level. We defined the response area from PSTHs showing the optimal response including those bins underlying the center response but not the secondary peaks (Jones and Sillito, 1994). Thus the LT index could vary from 0 (not length tuned) to 1 (no response to non-optimal, i.e., $100 \%$ length tuned); bar length $=0$ represents spontaneous activity.

\section{RESULTS}

Visual information from the retina reaches layer 4 of primary visual cortex indirectly via a relay in the LGN of the thalamus. After extensive laminar processing in primary visual cortex, the loop is closed by means of a dense feedback connection arising from pyramidal cells in layer 6 (Fig. 1). Although feedback mechanisms of processing are common to all neuronal systems, their precise role remains undetermined.

The results presented in this paper were obtained from 55 cells ( $28 \mathrm{X}, 24 \mathrm{Y}$, and 3 unclassified) recorded in the A laminas of the LGN of adult cats. We found no difference regarding LGN cell types.

\section{Effect of blocking cortical inputs mediated by metabotropic receptors on thalamic response properties}

\section{Effect of LY367385 on moving stimuli}

Thalamic and cortical cells differ not only in their receptive field structure but also in other response properties such as their sensitivity to moving versus static stimuli. To maximize the effect of blocking mGluR1 receptors, we used those stimuli that more strongly activate cortical cells. Figure 2 shows the response of an $\mathrm{X}$ ON LGN cell to a drifting grating of optimal spatial and

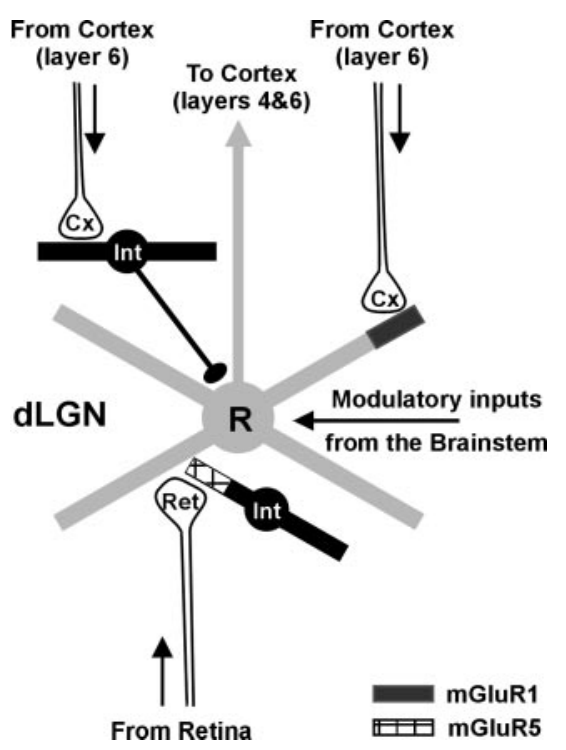

Figure 1. Basic circuitry of the cat dorsal lateral geniculate nucleus $(d L G N)$. Relay cells $(R)$ receive visual input from the retina (Ret) and send their axons to layers 4 and 6 of primary visual cortex $(C x)$. The loop is closed by a massive feedback projection from cortical layer 6 to the thalamus. Layer 6 axons contact the distal dendrites of relay cells, where they activate mGluR1. Blocking this particular type of metabotropic glutamate receptor specifically affects cortically mediated excitation because corticothalamic synapses onto interneurons (Int) are not mediated by mGluR1. Circuitry to and from the perigeniculate nucleus has been omitted for simplicity.

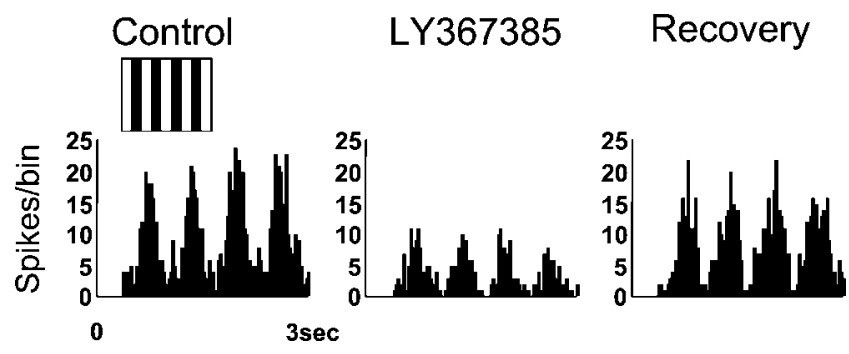

Figure 2. Blocking cortical input decreases LGN responses to moving stimuli. Data refer to an ON-center X cell. PSTHs illustrate the response of the cell to a full-field sinusoidal drifting grating (inset above the PSTH) of optimal characteristics before (Control), in the presence of the specific mGluR1 antagonist (ejected iontophoretically using $60 \mathrm{nA}$ for $6 \mathrm{~min}$ ), and after a recovery period of $15 \mathrm{~min}$. The histogram was constructed from 15 presentations of the stimulus. The representation of the stimulus has a merely graphic purpose and does not reflect its actual properties.

temporal frequency before, during, and after the ejection of LY367385. Blocking of mGluR1s significantly decreased the neuron response (by 64\%), which recovered to control levels after $\sim 15 \mathrm{~min}$. This result was found consistently in all cells examined (average diminution $\pm \mathrm{SD} ; 42 \pm 4 \% ; n=21$ ). Occasionally we tested the action of LY367385 on the responses evoked by gratings with orthogonal orientations. The observed effect showed no difference ( $n=4$; data not shown) regarding the orientation of the stimulus.

Other stimuli that effectively drive cortical and thalamic cells are moving bars. In addition, LGN cells (like some cortical cells) are selective for the bar length and are known as length-selective, end-stopped, or length tuned (LT). In the LGN, this property is highly dependent on the integrity of the visual cortex, and it is considered to be an emergent property of the geniculocortical 
Figure 3. The effect of LY367385 on the length tuning of LGN cells. Data are taken from an OFF-center $\mathrm{X}$ cell. Length-tuning curves were constructed for a bar of varying length moving in both directions over the receptive field. Bar width in this case was $0.5^{\circ}$. The bar lengths were presented in a interleaved randomized sequence. Responses were averaged over 15 trials and assessed from the accumulated count in the bins constituting the response area in the PSTH after subtraction of the background discharge. Black curve is control response; gray curve is response during LY367385 application (6 min, $60 \mathrm{nA}$ ). For each length of the bar, the corresponding PSTH is shown. It is clear that the response decreases when the metabotropic antagonist is ejected. The strongest effect is seen for the optimal length $\left(3^{\circ}\right.$ in this example).

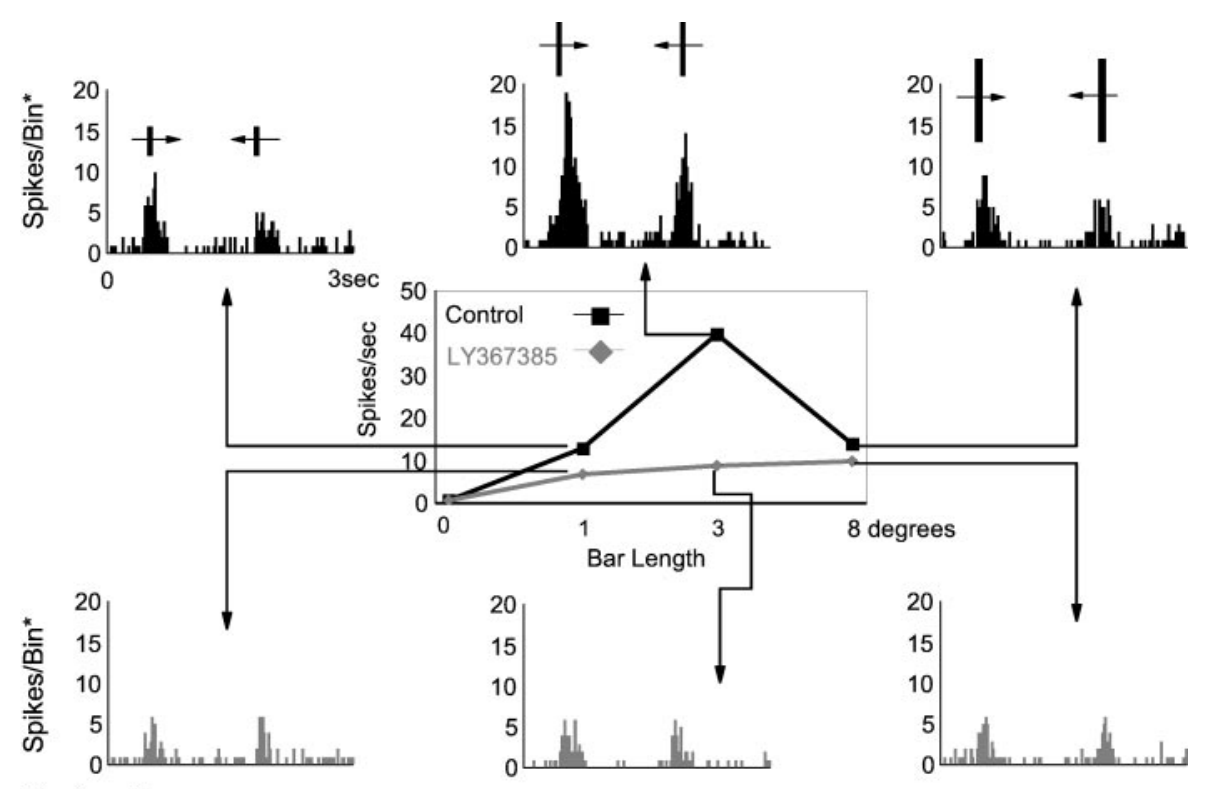

•Bin size $=25 \mathrm{msec}$
A

B

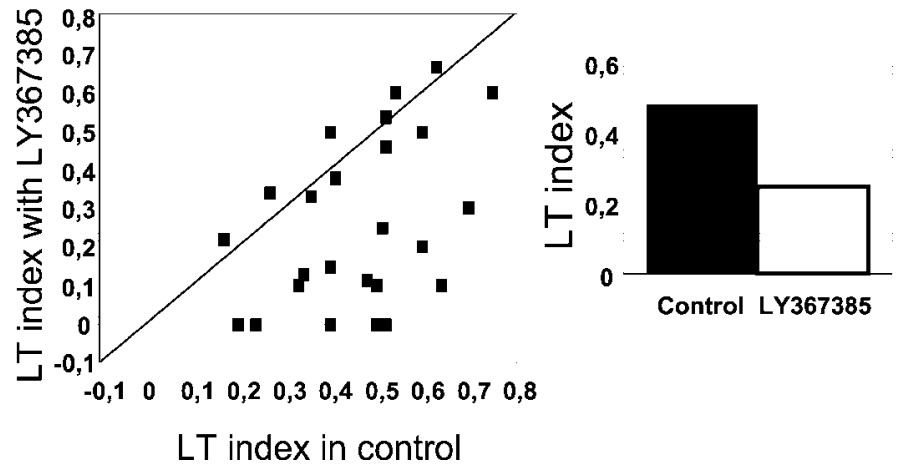

Figure 4. Length-tuning index in the LGN decreases with LY367385 ejection. $A$, Scatter plot comparing the LT index before and during blockade of mGluR1s by mean of LY376385 iontophoretic ejection $(n=$ $25)$. It is very evident that in a group of cells $(n=14)$, the LT decreases when the metabotropic antagonist is ejected (cells become less length tuned). $B$, Bar histogram showing the average LT in the two conditions for the cells represented in $A$.

loop (Murphy and Sillito, 1987). Figure 3 shows an example of a representative cell. In the center of the figure, the tuning curve illustrates the responses of the neuron during control conditions (black) and during ejection of LY367385 (gray). The histograms show a detailed response for each of the three selected lengths of the bar. During the control period, the cell shows the classical LT curve, the response reaching a maximum when the length of the bar fits the dimensions of the RF center ( $3^{\circ}$ in this example), declining in response to longer bars. After $6 \mathrm{~min}$ of continuous ejection of LY367385, the responses of the cell are reduced. However, it is important to note that the reduction affects most the responses to the optimal length. Recovery was obtained after 10 min (data not shown). The same result was obtained in $56 \%$ of the cells studied $(n=25)$. On average, the LT index (see Materials and Methods) decreased by $55 \%$. The scatter plot in Figure $4 A$ shows the LT index in control conditions against LT index during LY367385 ejection for all cells. In 14 cases the LT index clearly decreased during the mGluR1 blockade (Wilcoxon test; $p<0.01)$. In the remaining 11 cells there was a similar change to all stimuli, and therefore the LT index remained unaffected. The bar histogram in Figure $4 B$ represents the average decrease in LT index for the entire population $(n=25)$.

\section{Effect of LY367385 on static stimuli}

We have shown that metabotropic glutamate receptors are functionally active in the LGN in response to stimuli that optimally activate cortical cells. What happens with static stimuli? We examined the effect of LY367385 on the responses evoked by flashed spots covering the RF center. During the blockade of mGluR1 receptors, the response to the stimulus remains unaffected (Fig. $5 A$ ). In these cases, the response to flashing spots was not reduced, but when the presentation of the stimulus was long enough, we observed that the sustained phase of the response clearly decreased during LY367385 application (Fig. 5B) (notice that the transient phase of the response is not affected). This effect, and the concomitant reduction in spontaneous activity, suggests that the cortical feedback, mediated by mGluR1, plays an important role in regulating LGN excitability. In some cases, we did observe a slight diminution in the magnitude of the response, but this reduction was always much smaller than that obtained when moving stimuli were used. The average decrease was $9 \%$ $(n=25)$, clearly below the one obtained when moving bars of similar length were used. This small diminution in response could be a consequence of a reduction in excitability, because spontaneous activity was also reduced when mGluR1s were blocked $(56 \%)$. Interestingly, spontaneous activity did not decrease in $30 \%$ of the cells studied $(n=37)$.

\section{LY367385 does not produce an unspecific decrease in excitability}

The results presented above suggest that blocking mGluR1mediated cortical input reduces LGN excitability. This action is reflected in a decrease in the spontaneous activity and the sustained component of the response to long-lasting flashing spots. However, the effect of LY367385 on responses to moving stimuli suggests that the role of the cortical feedback is more complex than a global, unspecific modulation in firing rate as a result of, for instance, increased hyperpolarization. To further exclude this 

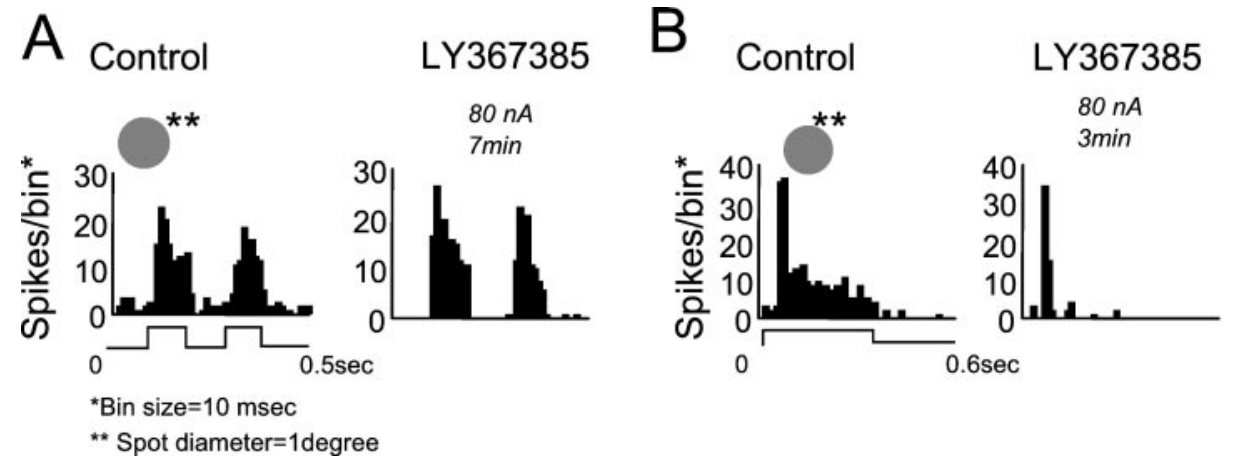

Recovery

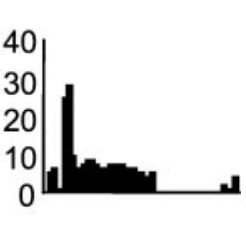

Figure 5. Transient responses are not affected by mGluR1 blockade. PSTHs showing the response of two LGN cells (ON X in $A$, OFF Y in $B)$ to flashed spots restricted to RF center (inset above the PSTH), before (Control), and in the presence of the specific mGluR1 antagonist. Duration of the stimuli was $0.1 \mathrm{sec}$ in $A$ and $0.3 \mathrm{sec}$ in $B$. LY367385 ejection: $80 \mathrm{nA}, 7 \mathrm{~min}$ in $A ; 80 \mathrm{nA}, 3 \mathrm{~min}$ in $B$. The representation of the stimulus has a merely graphic purpose and does not reflect its actual properties.
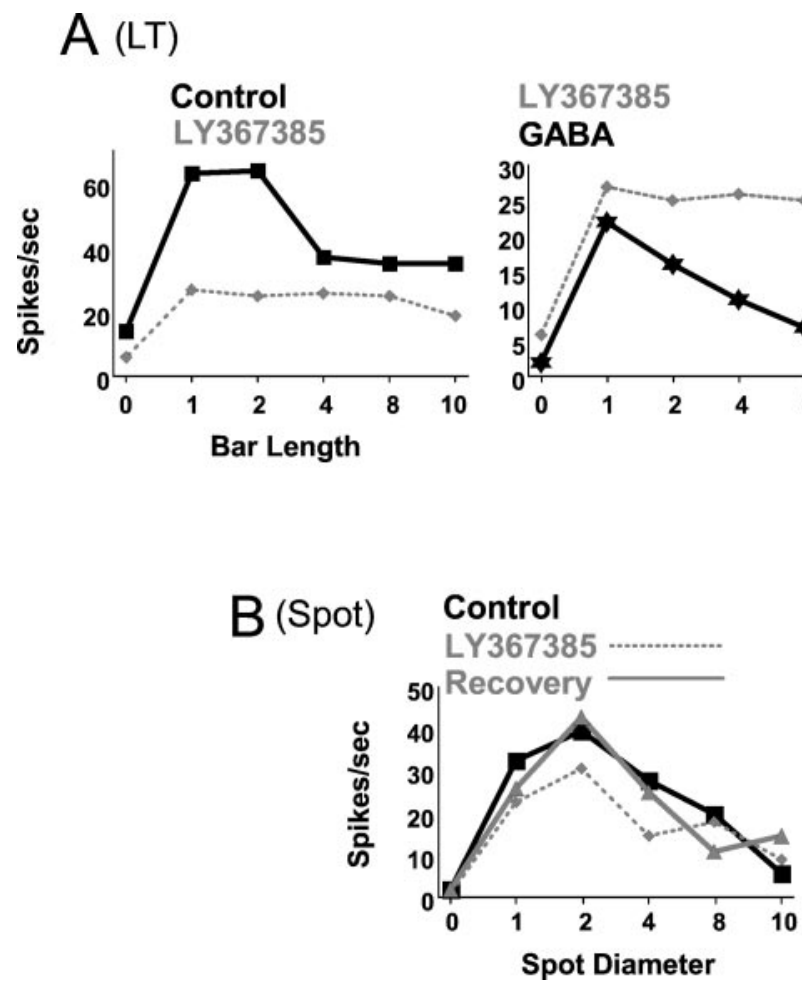

as a possibility, we directly compared the effects of LY367385 and GABA (the neurotransmitter responsible for inhibition in the LGN) on visual responses of six LGN cells. Figure $6 A$ shows results obtained from a $\mathrm{X}$ ON cell. In control conditions (Fig. $6 A$, left histogram, black line), the cell had an LT index of 0.47 . During LY367385 ejection (dotted line), selectivity to bar length was mostly abolished because the response to the optimal stimulus was strongly affected. During GABA application (Fig. 6A, center histogram, black line), visual responses markedly decreased to all stimuli (note the different $y$-axis scale). However, the cell clearly maintained selectivity for stimulus length. Moreover, the response of this cell to flashing spots of increasing diameters was not affected by LY367385 (Fig. 6B).

\section{Effect of LY367385 on the organization of LGN receptive fields}

A fundamental property of LGN receptive fields is the centersurround antagonism. This attribute is retinal in origin, but it has
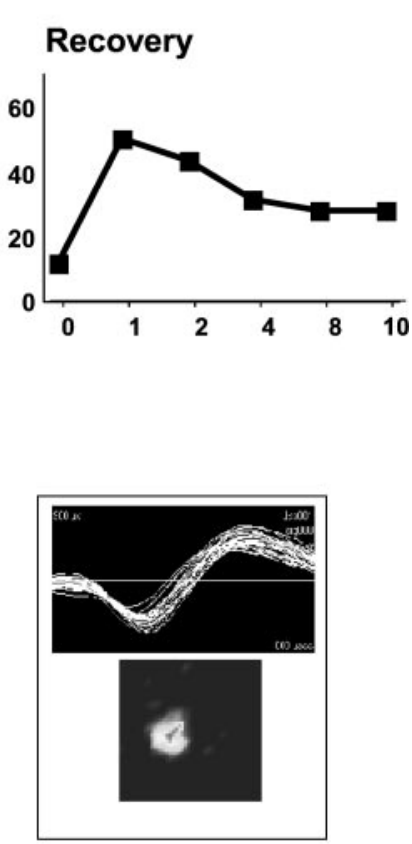

Figure 6. The observed effect is not caused by an unspecific decrease in excitability. Results are from an ON X cell. $A$, Length-tuning curve showing the response of the cell in different conditions: control versus LY367385 ejection (left), LY367385 versus GABA (center; notice the different scale in the $y$-axis), and recovery (right). $B$, Spot diameter tuning curve for the same cell in control, during LY367385, and recovery. Waveform and RF structure (in a $10 \times 10^{\circ}$ grid) are shown in the inset on the right.

been shown to be influenced by visual cortex (Murphy and Sillito, 1987; Cudeiro and Sillito, 1996). To study the effect of blocking mGluR1 receptors on the basic organization of the thalamic receptive field, we followed two strategies. First, we studied the effect of LY367385 application on the degree of center-surround antagonism measured by comparing responses to flashed spots of two different sizes: one just covering the receptive-field excitatory center and the other covering both center and surround. Figure 7 illustrates the results obtained from a Y ON cell. Before the application of LY367385 (black), the cell showed rather strong center-surround antagonism, i.e., the optimal response was obtained for a stimulus size $\left(3^{\circ}\right)$ that matched the size of the receptive-field center (top histogram). When the size of the spot was increased to $8^{\circ}$, covering simultaneously center and surround, the response dropped markedly (bottom histogram). This effect was detected in both the transient and sustained phases of the response. When mGluR1s were blocked (Fig. 7, gray), the tran- 

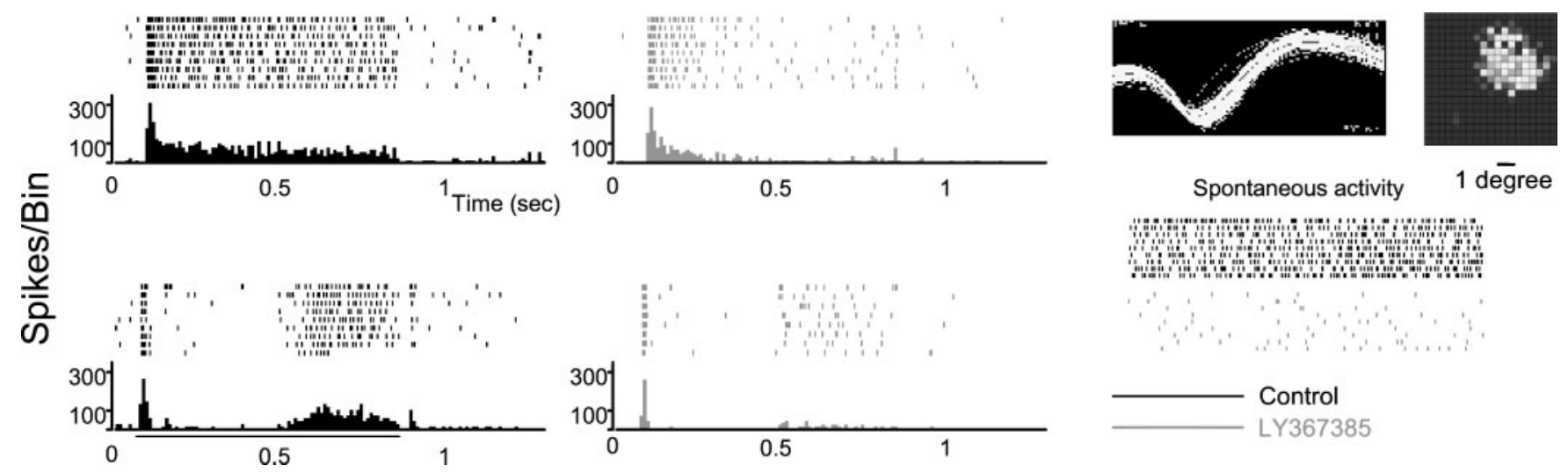

Figure 7. Effect of LY367385 application on the center-surround organization of LGN receptive fields. PSTHs and raster plots show the response of an ON Y cell to a flashing spot restricted to the RF center (top) and covering center and surround (bottom) in control (black) and during LY367385 ejection ( gray). The waveform and RF obtained with sparse noise and the effect of blocking mGluR1 on spontaneous activity are shown on the right.
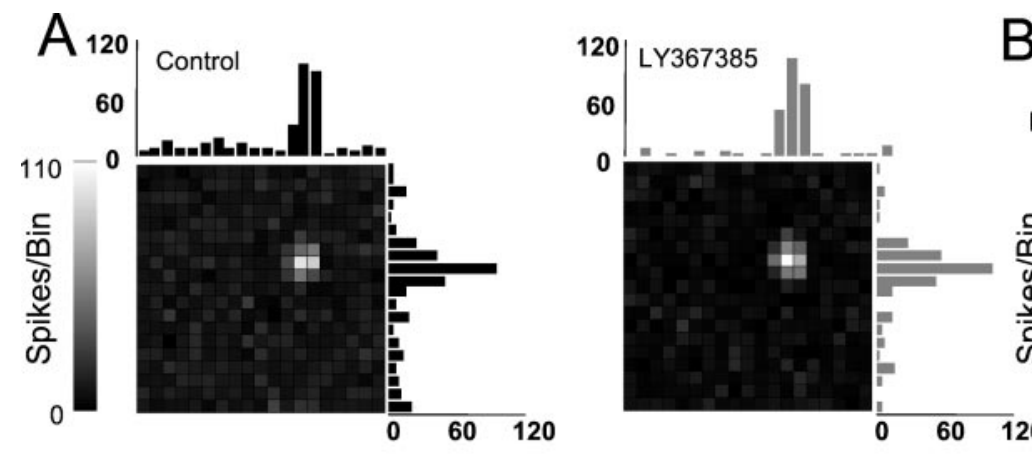

B

Response at the peak of the RF center

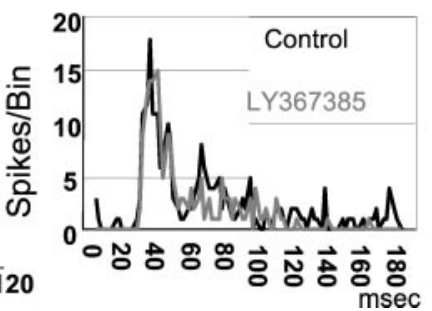

Figure 8. mGluR1 blockade does not affect the spatiotemporal structure of the RF. $A$, RF map $\left(10 \times 10^{\circ}\right)$ obtained with sparse noise for an ON X cell in control (left) and during mGluR1 blockade. Bar histograms represent the responses obtained through a line crossing the RF center in the $x$ - and $y$-axis. Grayscale bar is for both maps. $B$, Impulse response at the peak of the RF center in the two conditions. Bin $=2$ msec.

sient response to the small and large spots was conserved, but the sustained component was clearly affected in both cases.

The second approach consisted of using a sparse noise protocol (see Materials and Methods) to obtain a precise representation of the spatiotemporal structure of the RF center. Figure $8 A$ shows the RF of an X ON cell, obtained at 20-60 msec, before and during LY367385 application. The sparse noise consisted of white spots flashed on a dark background (contrast, 100\%). The bar histograms next to the pixel maps represent the response of the cell to stimuli that fell on pixels located along two imaginary lines crossing the RF center in the $x$ - and $y$-axis. The only difference between the two maps is a decrease in the background activity during the blockade of mGluR1, visible as a darker background in the map and a lower level in the baseline of the histograms. Figure $8 B$ shows that neither the time course of the response at the peak of the receptive field nor its spatial structure were affected during LY367385 application (gray line). Although the stimulus was updated every $40 \mathrm{msec}$, spike counts were taken in 2 msec bins to precisely check potential changes in latency, duration, or magnitude of the response.

\section{DISCUSSION}

LY367385 is a highly specific mGluR1 antagonist (Clark et al., 1997; Marino et al. 2001), and it has previously been shown in vivo that this compound does not affect responses mediated by either other metabotropic or ionotropic glutamate receptors (Salt et al.,
1999). Furthermore, there is clear evidence that the corticof ugal axons are the only source of synaptic input to the LGN, which uses mGluR1 (Godwin et al., 1996). Given these data, we believe that the results reported in this paper are therefore the direct consequence of modifying the corticothalamic input mediated by metabotropic receptors.

Here we have demonstrated that $\sim 60 \%$ of LGN neurons, in the presence of the specific mGluR1 antagonist LY367385, decrease their selectivity for the length of effective moving bar stimuli. Specifically, this property is lost because the response to the optimal stimulus is strongly reduced, rather than there being an increase in response magnitude to longer stimuli. Second, this effect does not correlate with changes in the spatiotemporal structure of the RF as measured with a sparse noise protocol. Third, spontaneous firing is reduced. On the other hand, responses to simple, static stimuli, like flashed spots, remain largely unaffected when the mGluR1 antagonist LY367385 is applied. However, when the presentation of the stimulus is long enough to evoke a sustained response, this phase of the response is notably reduced.

The most parsimonious interpretation of our results is that mGluR1-mediated cortical input modulates LGN responses via two different mechanisms acting simultaneously. First, it would modulate excitability, as indicated by the decrease in spontaneous activity when ejecting LY367385. Probably this is the result of withdrawing some tonic component of the excitatory input from 
cortex to LGN that is mediated by mGluR1s (Turner and Salt, 2000).

A second mechanism would specifically enhance thalamic responses to those stimuli that strongly activate cortical neurons. We have demonstrated that a simple hyperpolarization, such as that induced by local application of GABA, affected the responses to all the stimuli by a similar amount, without affecting the LT of the cells. Hence, the effect of LY367385 on thalamic responses cannot be explained solely on the basis of a nonspecific hyperpolarization, but rather requires that the normal excitatory drive is "permissive" specifically to the geniculate expression of its excitatory retinal input, revealed when effective cortical stimuli are used.

The role of the corticothalamic input on thalamic response properties has been a matter of intense work, both in vivo and in vitro, during the past several years (Murphy and Sillito, 1987; McCormick and von Krosigk, 1992; Sillito et al., 1994; Marrocco et al., 1996; Murphy et al., 1999; Bal et al., 2000). Previous experimental approaches included suppressing cortical input by surgically removing or cooling the cortex, pharmacological modulation of cortical activity, etc. Our experimental design, which was based on two recent findings- the discovery that at the level of the LGN, mGluR1s are restricted to corticothalamic synapses onto relay cells, and the development of an antagonist specific for mGluR1-has two major advantages: it restricts the extent of the blockade, and it recovers in a reasonable time, which allowed us to test the response of the same cell in several conditions.

\section{Effects on moving stimuli}

The main effect described in this paper is a decrease in thalamic LT when cortical input is blocked. This decrease in bar-length selectivity is not produced by an increase in the response to the non-optimal stimuli, as demonstrated by Sillito and colleagues (Murphy and Sillito, 1987), but rather by a specific reduction of the response to the optimal stimulus. Murphy and Sillito (1987) reported a loss of LT in LGN cells when visual cortex was surgically removed, eliminating all cortical input to the thalamus. This loss of LT was the result of increased responses to longer stimuli, indicating that length tuning was the result of cortically driven geniculate inhibition. In the data above we reveal a second component, by locally suppressing part of the excitatory cortical input (mediated by mGluR1 receptors) without directly affecting cortically driven inhibition [cortical terminals activate only ionotropic receptors of LGN interneurons (Pape and McCormick, 1995)]. To account for the changes in LT that we show here, we suggest that this mGluR1-mediated corticof ugal excitation should be insufficient on its own to provide an effective drive for the LGN center. Rather, the more weakly length-tuned retinal input must summate, probably nonlinearly, with the cortical drive to create the center response to moving stimuli. As a result, if we remove the cortical drive, the response at the center collapses, producing a distortion in length summation (flattening the lengthtuning curve).

Our findings fit nicely with recent data obtained in the somatosensory system by Canedo and Aguilar (2000), who showed that cortical input enhances thalamic and cuneate responses produced by stimuli located at the center of the RF. Our data seem to indicate that visual cortex modulates thalamic activity to promote information transfer through specific locations in sensory space. Because we are locally blocking mGluR1, we cannot rule out the possibility that removing cortical inputs at increasing distances in the LGN would further modify thalamic response properties, particularly to those stimuli that specifically engage the surround of the receptive field. Importantly, a significant percentage of cells in our sample (11 of 25 ) did not show a reduction in LT index in the presence of LY367385; rather, responses to stimuli of all sizes were reduced by a similar amount. To a first approximation, our sample then seems to comprise cells that belong to two separate classes of LGN neurons distinctly affected by the cortical input. However, none of the tests performed systematically to characterize all recorded cells allowed us to detect further consistent differences between the two cell types. Thus both groups contained a similar proportion of X and Y cells. Moreover, cells belonging to the two groups were found during the same penetration, with no trace of clustering according to laminar location or distance from the area centralis. An attractive possibility is that the two groups represent relay cells and interneurons. On the basis of their responses to iontophoretical ejection of Ach (McCormick and Prince, 1987), we conclude that most of our recordings are from relay cells. One could argue that the two groups represent two novel cell classes in the LGN. However, because we are locally blocking $\mathrm{mGluR} 1$ receptors using iontophoretic application of the drug, we cannot discard the possibility that more effective removal of cortical inputs at different distances in the LGN (by increased ejection currents, longer durations, etc.), or even a differential distribution of such inputs on a given dendritic tree, would further modify thalamic response properties.

\section{Effects on static stimuli}

Cortical feedback has been related to changes in the structure, and even the position, of LGN receptive fields (Wang et al., 2000). Our results using sparse noise, however, show that blocking mGluR1-mediated cortical input has no effect on the spatiotemporal structure of thalamic receptive fields. This apparent disagreement could arise from two important methodological differences. First, Wang et al. (2000) topically applied bicuculline to the cortex, which increases cortical input over a large area of LGN. Second, our experimental approach does not directly affect cortically driven inhibitory events in the LGN, which may "shape" the RF dynamically. Whether or not the spatial structure of each component of the field changes in the presence of LY367385, stronger effects would be expected on responses to stimuli that do induce complex center-surround interactions, like concentric stimuli with different orientations (Sillito et al., 1993).

Blocking mGluR1 reduced spontaneous activity in $70 \%$ of the cells recorded. This is in good agreement with previous reports by Tsumoto et al. (1978) showing that cortical input to LGN is excitatory when it comes from cortical regions in good retinotopic alignment. Furthermore, mGluR1-mediated cortical input has been shown to evoke a long-lasting excitatory postsynaptic potential from the reduction of a potassium conductance (McCormick and von Krosigk, 1992) that is associated with an increased neuronal excitability (Turner and Salt, 2000). This excitatory effect could explain the observed reduction in the sustained responses when mGluR1s are blocked.

Our results suggest that cortical feedback, mediated by mGluR1, modulates center-surround interactions by specifically enhancing the responses at the center of the field. In addition, results by others have demonstrated cortically mediated lateral inhibition in the LGN (Tsumoto et al., 1978; Murphy and Sillito, 1987). Thus the corticothalamic projection, using a push-pull mechanism that selectively enhances center-excitation or surround-inhibition, or both, may be capable of modulating thalamic activity to promote transfer through specific locations in 
sensory space. Furthermore, the time scale of the metabotropic corticothalamic EPSP is on the order of seconds (McCormick and von Krosigk, 1992) and might be implicated in different plastic processes, mediating experience-dependent changes in thalamic responses (Cudeiro et al., 2000). In the early visual system, feedforward connections shape the classical receptive field (e.g., from the retina to LGN to V1) (Alonso et al., 1996; Tavazoie and Reid, 2000), and by means of feedback pathways, neurons receive information form the surround. Here we have shown that by acting on metabotropic receptors, feedback connections reinforce the center of the RF and hence the salience of the message passed to the visual cortex.

\section{REFERENCES}

Ahlsen G, Lo FS (1982) Projection of brain stem neurons to the perigeniculate nucleus and the lateral geniculate nucleus in the cat. Brain Res 238:433-438.

Alonso JM, Usrey WM, Reid RC (1996) Precisely correlated firing in cells of the lateral geniculate nucleus. Nature 383:815-819.

Bal T, Debay D, Destexhe A (2000) Cortical feedback controls the frequency and synchrony of oscillations in the visual thalamus. J Neurosci 20:7478-7488.

Bickford ME, Gunluk AE, Guido W, Sherman SM (1993) Evidence that cholinergic axons from the parabrachial region of the brainstem are the exclusive source of nitric oxide in the lateral geniculate nucleus of the cat. J Comp Neurol 334:410-430.

Canedo A, Aguilar J (2000) Spatial and cortical influences exerted on cuneothalamic and thalamocortical neurons of the cat. Eur J Neurosci 12:2515-2533.

Clark BP, Baker SR, Goldsworthy J, Harris JR, Kingston AE (1997) 2-Methyl-4-carboxyphenylglycine (LY367385) selectively antagonizes metabotropic glutamate mGluR1 receptors. Bioorg Med Chem Lett 7:2777-2870.

Cudeiro J, Rivadulla C (1999) Sight and insight: on the physiological role of nitric oxide in the visual system. Trends Neurosci 22:109-116.

Cudeiro J, Sillito AM (1996) Spatial frequency tuning of orientationdiscontinuity-sensitive corticofugal feedback to the cat lateral geniculate nucleus. J Physiol (Lond) 490:481-492.

Cudeiro J, Rivadulla C, Grieve KL (2000) Visual response augmentation in cat (and macaque) LGN: potentiation by corticof ugally mediated gain control in the temporal domain. Eur J Neurosci 12:1135-1144.

De Lima AD, Singer W (1987a) The serotoninergic fibers in the dorsal lateral geniculate nucleus of the cat: distribution and synaptic connections demonstrated with immunocytochemistry. J Comp Neurol 258:339-351.

De Lima AD, Singer W (1987b) The brainstem projection to the lateral geniculate nucleus in the cat: identification of cholinergic and monoaminergic elements. J Comp Neurol 259:92-121.

Erisir A, Van Horn SC, Bickford ME, Sherman SM (1997) Immunocytochemistry and distribution of parabrachial terminals in the lateral geniculate nucleus of the cat: a comparison with corticogeniculate terminals. J Comp Neurol 377:535-549.

Godwin DW, Van Horn SC, Eriir A, Sesma M, Romano C, Sherman SM (1996) Ultrastructural localization suggests that retinal and cortical inputs access different metabotropic glutamate receptors in the lateral geniculate nucleus. J Neurosci 16:8181-8192.

Hirsch JA, Alonso JM, Reid RC, Martinez LM (1998) Synaptic integration in striate cortical simple cells. J Neurosci 18:9517-9528.

Hubel DH, Wiesel TN (1965) Receptive fields and functional architecture in two non-striate visual areas (18 and 19) of the cat. J Neurophysiol 28:229-287.

Jones HE, Sillito AM (1994) The length-response properties of cells in the feline perigeniculate nucleus. Eur J Neurosci 6:1199-1204.

Jones JP, Palmer LA (1987). The two-dimensional spatial structure of simple receptive fields in cat striate cortex. J Neurophysiol 58:1187-1211.

Marino MJ, Wittmann M, Bradley SR, Hubert GW, Smith Y, Conn PJ (2001) Activation of group I metabotropic glutamate receptors produces a direct excitation and disinhibition of GABAergic projection neurons in the substantia nigra pars reticulata. J Neurosci 21:7001-7012.

Marrocco RT, McClurkin JW, Alkire MT (1996) The influence of the visual cortex on the spatiotemporal response properties of lateral geniculate nucleus cells. Brain Res 737:110-118.

McCormick DA, Prince DA (1987) Actions of acetylcholine in the guinea-pig and cat medial and lateral geniculate nuclei, in vitro. J Physiol (Lond) 392:147-165.

McCormick DA, von Krosigk M (1992) Corticothalamic activation modulates thalamic firing through glutamate "metabotropic" receptors. Proc Natl Acad Sci USA 89:2774-2778.

Montero VM (1986) Localization of gamma-aminobutyric acid (GABA) in type 3 cells and demonstration of their source to F2 terminals in the cat lateral geniculate nucleus: a Golgi-electron-microscopic GABAmmunocytochemical study. J Comp Neurol 254:228-245.

Montero VM (1987) Ultrastructural identification of synaptic terminals from the axon of type 3 interneurons in the cat lateral geniculate nucleus. J Comp Neurol 264:268-283.

Montero VM, Wenthold RJ (1989) Quantitative immunogold analysis reveals high glutamate levels in retinal and cortical synaptic terminals in the lateral geniculate nucleus of the macaque. Neuroscience 31:639-647.

Murphy PC, Sillito AM (1987) Corticofugal feedback influences the generation of length tuning in the visual pathway. Nature 329:727-729.

Murphy PC, Duckett SG, Sillito AM (1999) Feedback connections to the lateral geniculate nucleus and cortical response properties. Science 286:1552-1554.

Pape H-C, McCormick DA (1995) Electrophysiological and pharmacological properties of interneurons in the cat dorsal lateral geniculate nucleus. Neuroscience 68:1105-1125.

Salt TE, Turner JP, Kingston AE (1999) Evaluation of agonists and antagonists acting at group I metabotropic glutamate receptors in the thalamus in vivo. Neuropharmacology 38:1505-1510.

Scharfman HE, Lu SM, Guido W, Adams PR, Sherman SM (1990) $N$-methyl-D-aspartate receptors contribute to excitatory postsynaptic potentials of cat lateral geniculate neurons recorded in thalamic slices. Proc Natl Acad Sci USA 87:4548-4552.

Sillito AM, Cudeiro J, Murphy PC (1993) Orientation sensitive elements in the corticofugal influence on center-surround interactions in the dorsal lateral geniculate nucleus. Exp Brain Res 93:6-16.

Sillito AM, Jones HE, Gerstein GL, West DC (1994) Feature-linked synchronization of thalamic relay cell firing induced by feedback from the visual cortex. Nature 369:479-482.

Singer W (1977) Control of thalamic transmission by corticofugal and ascending reticular pathways in the visual system. Physiol Rev 57:386-420.

Steriade M, Pare D, Parent A, Smith Y (1988) Projections of cholinergic and non-cholinergic neurons of the brainstem core to relay and associational thalamic nuclei in the cat and macaque monkey. Neuroscience 25:47-67.

Tavazoie SF, Reid RC (2000) Diverse receptive fields in the lateral geniculate nucleus during thalamocortical development. Nat Neurosci 3:608-616.

Tsumoto T, Creutzfeldt OD, Legendy CR (1978) Functional organization of the corticofugal system from visual cortex to lateral geniculate nucleus in the cat (with an appendix on geniculo-cortical mono-synaptic connections). Exp Brain Res 32:345-364.

Turner JP, Salt TE (2000) Synaptic activation of the group I metabotropic glutamate receptor mGlu1 on the thalamocortical neurons of the rat dorsal lateral geniculate nucleus in vitro. Neuroscience 100:493-505.

Uhlrich DJ, Tamamaki N, Murphy PC, Sherman SM (1995) Effects of brain stem parabrachial activation on receptive field properties of cells in the cat's lateral geniculate nucleus. J Neurophysiol 73:2428-2447.

Updyke BV (1975) The patterns of projection of cortical areas 17, 18, and 19 onto the laminae of the dorsal lateral geniculate nucleus in the cat. J Comp Neurol 163:377-396.

Updyke BV (1977) Topographic organization of the projection from cortical areas 17, 18, and 19 onto the thalamus, pretectum and superior colliculus in the cat. J Comp Neurol 173:81-122.

Van Horn SC, Erisir A, Sherman SM (2000) Relative distribution of synapses in the A-laminae of the lateral geniculate nucleus of the cat. J Comp Neurol 416:509-520.

Varela C, Rivadulla C, Martinez L, Cudeiro J (2000) Stimulusdependent modulation of LGN activity by cortically activated metabotropic glutamate receptors. Soc Neurosci Abstr 26:1197.

Wang W, Andolina IM, Jones HE, Salt TE, Sillito AM (2000) Influence of enhanced feedback from V1 on visual responses in the LGN. Soc Neurosci Abstr 26:447.

Wilson JR, Friedlander MJ, Sherman SM (1984) Fine structural morphology of identified X- and Y-cells in the cat's lateral geniculate nucleus. Proc R Soc Lond B Biol Sci 221:411-436. 